\title{
Widespread Projections from Myelinated Nociceptors throughout the Substantia Gelatinosa Provide Novel Insights into Neonatal Hypersensitivity
}

\author{
C. Jeffery Woodbury and H. Richard Koerber \\ Department of Neurobiology, University of Pittsburgh School of Medicine, Pittsburgh, Pennsylvania 15261
}

Skin sensory neurons have long been thought to undergo major changes in anatomy and physiology over the first few weeks of postnatal life. Low-threshold mechanoreceptors (LTMRs) are believed to project extensively throughout superficial dorsal horn laminas initially and provide the afferent limb for hyperactive nocifensive reflexes. However, our recent studies revealed that neonatal LTMRs do not project into "pain-specific" regions; instead, they exhibit adult-like anatomy shortly after birth. We sought to determine whether the same might be true for myelinated high-threshold mechanoreceptors (HTMRs). We used an intact, ex vivo somatosensory system preparation from neonatal mice to allow intrasomal recording and neurobiotin labeling of individual sensory neurons characterized via natural skin stimuli. Neonatal HTMRs displayed a number of key hallmarks of their adult counterparts; relative to LTMRs, they exhibited broader, inflected somal spikes and higher mechanical thresholds and/or responded in an increasingly vigorous manner to incrementally graded forces in a manner capable of encoding stimulus intensity. Two types were discerned on the basis of central anatomy: one subset projected to superficial laminas (I/II); the other gave rise to diffuse, dorsally recurving collateral arbors extending throughout the entire dorsal horn (I-V). The latter represent a novel cutaneous afferent morphology that persists in older animals. These studies reveal that inputs from myelinated afferents to superficial pain-specific laminas in neonates arise from HTMRs and not LTMRs as commonly thought. This frequently overlooked population is in a position, therefore, to contribute substantially to paradoxical nocifensive behaviors in neonates and various pain states in adults.

Key words: pain; development; spinal cord; dorsal horn; lamina II; skin; sensory; dorsal root ganglia; DRG

\section{Introduction}

Skin sensory neurons display a striking diversity of physiological and anatomic properties that are all tightly correlated with function in adults (for review, see Scott, 1992). Among myelinated afferents, for example, high-threshold mechanoreceptors (HTMRs, or nociceptors) exhibit broad somal action potentials with a characteristic inflection on the falling phase, in marked contrast to the narrow, uninflected somal spikes of low-threshold mechanoreceptors (LTMRs) (Rose et al., 1986; Koerber et al., 1988; Djouhri et al., 1998). The central processes of HTMRs and LTMRs also exhibit generally nonoverlapping terminations in the dorsal horn $(\mathrm{DH})$, with HTMRs projecting to both superficial and deeper laminas (i.e., I/IIo and V), whereas LTMRs terminate deep to superficial laminas (e.g., IIi-V) (for review, see Fyffe, 1992).

This tight correlation among anatomic and physiological properties is thought to occur over a protracted postnatal period. Thus, although all major functional classes of skin sensory neurons seem to be present in newborns (Fitzgerald, 1987), charac-

Received Aug. 26, 2002; revised 0ct. 23, 2002; accepted 0ct. 24, 2002.

This work was supported by National Institutes of Health Grant NS23725 (H.R.K.). C.J.W. was supported by National Institutes of Health Grant NS44094. We thank Dr. Amy M. Ritter for helpful suggestions, Corinne Batchelor, Julie Kopczak, and Weiwen Wang for excellent technical assistance, and Dr. Brian M. Davis and two anonymous reviewers for constructive comments on an earlier version of this manuscript.

Correspondence should be addressed to Dr. C. Jeffery Woodbury, Department of Neurobiology, University of Pittsburgh School of Medicine, W1413 Biomedical Science Tower, Pittsburgh, PA 15261. E-mail: woodbury+@pitt.edu.

Copyright $\odot 2002$ Society for Neuroscience $\quad 0270-6474 / 02 / 220601-10 \$ 15.00 / 0$ teristic somal spike and peripheral response properties have been suggested to mature later (Fulton, 1987; Fitzgerald and Fulton, 1992; Koltzenburg et al., 1997; Mirnics and Koerber, 1997; Ritter et al., 2000). Likewise, a significant delay in the development of central termination patterns among myelinated afferents has also been suggested. For example, LTMRs are thought to project inappropriately into superficial "pain-specific" DH laminas during the first few weeks of postnatal life (Fitzgerald et al., 1994; Coggeshall et al., 1996; Mirnics and Koerber, 1997) (but see Woodbury et al., 2000), thereby transiently serving as the afferent limb of hypersensitive nocifensive reflexes (for review, see Fitzgerald and Jennings, 1999).

Recent studies of individual, physiologically identified cutaneous afferents in newborn mice, however, reveal that this scenario of delayed maturity does not apply to LTMRs. Indeed, neonatal LTMRs are essentially miniaturized versions of their adult counterparts: they arborize in the same regions of the neonatal $\mathrm{DH}$ as they do in adults, exhibit stereotypical (i.e., adult-like) response properties, and display narrow, uninflected somal spikes (Woodbury et al., 2001). Because the latter findings also contradict the view that broad, inflected somal spikes represent an immature archetype among skin sensory neurons, one that is transcended postnatally only by LTMRs (for review, see Fitzgerald and Fulton, 1992; Koerber and Mendell, 1992), a key question is whether broad, inflected somal spikes signify a nociceptive phenotype in neonates as in adults.

Unlike LTMRs, few studies have been conducted on the spinal terminations of HTMRs (Light and Perl, 1979; Rethelyi et al., 
1982), and currently nothing is known of their development. Thus, the present studies examined the postnatal development of incipient myelinated HTMRs using an ex vivo somatosensory system preparation in mice, with spinal cord and skin in continuity, which allows combined analyses of somal spikes, peripheral response properties, and anatomy of individual afferents. The present data show that myelinated HTMRs are well developed shortly after birth. Unlike LTMRs, they give rise to widespread projections throughout superficial DH laminas and thus probably serve as the myelinated afferent limb of nocifensive behaviors.

\section{Materials and Methods}

The in vitro electrophysiological experiments described in the present report were conducted on early postnatal mice (Swiss-Webster strain; Hilltop Farms, Scottdale, PA) ranging in age from the day of birth [postnatal day $0(\mathrm{P} 0)$ ] to $\mathrm{P} 7$; experiments were also conducted on mice older than P7 for comparison. All procedures used in the present studies were approved by the University of Pittsburgh Animal Care and Use Committee.

Preparation and recording. The ex vivo cutaneous somatosensory system preparation used in the present studies was detailed previously (Woodbury et al., 2001). Briefly, neonates at P7 or younger were anesthetized with ice [mice older than P7 were anesthetized via intramuscular injection of ketamine and xylazine (90 and $10 \mathrm{mg} / \mathrm{kg}$, respectively)]. Anesthetized animals were quickly decapitated after transcardial perfusion with oxygenated $\left(95 \% \mathrm{O}_{2}-5 \% \mathrm{CO}_{2}\right)$ artificial CSF (aCSF) at $12-$ $15^{\circ} \mathrm{C}$ in which sodium chloride had been substituted with sucrose at equivalent osmolarity (in mM: 253.9 sucrose, $1.9 \mathrm{KCl}, 1.2 \mathrm{KH}_{2} \mathrm{PO}_{4}, 1.3$ $\mathrm{MgSO}_{4}, 2.4 \mathrm{CaCl}_{2}, 26.0 \mathrm{NaHCO}_{3}$, and $10.0 \mathrm{D}$-glucose). The spinal cord, thoracic and upper lumbar dorsal root ganglia (DRGs), dorsal cutaneous nerves (DCNs), and dorsolateral trunk skin on one side were dissected in continuity in a circulating bath of the same solution. Once isolated, this ex vivo preparation was transferred to a separate recording chamber through which chilled oxygenated aCSF, now replenished with sodium chloride (in mm: $127.0 \mathrm{NaCl}, 1.9 \mathrm{KCl}, 1.2 \mathrm{KH}_{2} \mathrm{PO}_{4}, 1.3 \mathrm{MgSO}_{4}, 2.4 \mathrm{CaCl}_{2}$, $26.0 \mathrm{NaHCO}_{3}$, and $10.0 \mathrm{D}$-glucose), was circulated. The preparation was pinned out with the epidermal surface of the skin facing upward, and the recording bath was raised to $30-31^{\circ} \mathrm{C}$ for electrophysiological recording.

Individual sensory neuron somata were impaled in the DRGs with glass microelectrodes $(>100 \mathrm{M} \Omega$ ) containing 5-20\% neurobiotin (Vector Laboratories, Burlingame, CA) in $1 \mathrm{~m}$ potassium acetate. Electrical search stimuli were delivered through a suction electrode on the nerve to locate afferents with a peripheral process in the DCNs. Peripheral receptive fields (RFs) were located via gentle stimulation with a blunt glass stylus, after which calibrated von Frey filaments were used to characterize the mechanical threshold of the neuron and its response properties to graded mechanical stimuli (these filaments provide a discontinuous series of increasing forces and may not reveal small differences in threshold or sensitivity). Evoked activity was digitized on tape for subsequent offline analyses. After physiological characterization, the cell was stained by injecting neurobiotin iontophoretically (one per DRG). Peripheral conduction velocity $(\mathrm{CV})$ was calculated from spike latency and the distance between stimulating and recording electrodes (measured directly along the nerve).

Afferent identification. The primary goal of these studies was to examine myelinated afferents falling under the general definition of HTMRs, i.e., myelinated afferents responding selectively to tissue-damaging stimulus intensities and those providing an increasingly vigorous response to increasing stimulus intensities and thereby capable of warning against potential damage (Burgess and Perl, 1967). However, most afferents conduct $<1 \mathrm{~m} / \mathrm{sec}$ at these ages (Woodbury et al., 2001). Toward the first goal, because of changing myelination state during the early postnatal period (Ziskind-Conhaim, 1988) and observed variability in growthmaturational rate between animals of similar age (a consequence of differing litter sizes), relative latency measures of individual DCN afferents were made on a nerve-by-nerve basis. That is, to sample the broad spectrum of future myelinated afferents, all but the most slowly conducting group of sensory neurons were targeted in any given nerve. The latter, constituting the most frequently encountered afferents throughout these experiments, consistently exhibited broad, inflected somal action potentials and, for any given nerve, relatively uniform latencies. Their calculated CVs correlated with the slowest of multiple peaks seen in a number of compound action potential recordings (data not shown). Far more definitively, however, many were stained for independent verification of their identity, and all exhibited highly stereotypical central terminations indicative of afferents that will remain unmyelinated (i.e., future C-fiber afferents) (Woodbury et al., 2001, their Fig. 7); hence, although the possibility remains that some of the most slowly conducting future myelinated afferents were missed by this approach, it is highly probable, particularly in light of findings from central anatomy (see Results), that all neurons included in the present study represent future (i.e., incipient) myelinated afferents.

To achieve the second goal, because adult myelinated HTMRs span an extremely broad spectrum of mechanical thresholds, CVs, adaptation properties, and somal spike properties (Burgess and Perl, 1967; Koerber et al., 1988), no single property in isolation (e.g., mechanical threshold) can adequately diagnose these afferents in mature animals, much less among neonates, in which the parameters of these various properties are currently unknown. Therefore, the approach taken was to examine afferents satisfying a combination of a number of different properties that represent key hallmarks of adult myelinated HTMRs. Because neonatal LTMRs and C-fiber afferents exhibit adult-like somal spikes (Woodbury et al., 2001) and because of the possibility that mechanical thresholds of HTMRs may be lower in neonates than adults (Ritter et al., 2000), the primary focus was on incipient myelinated afferents exhibiting (1) inflected somal spikes, (2) slowly adapting (SA) response properties, (3) elevated mechanical thresholds, and/or (4) an increasingly vigorous response to increasing forces, as noted for adult HTMRs.

Tissue processing and analysis. Two to $6 \mathrm{hr}$ after neurobiotin iontophoresis, the spinal cord was immersion fixed [ $4 \%$ paraformaldehyde in $0.1 \mathrm{~m}$ phosphate buffer, $\mathrm{pH} 7.4(\mathrm{~PB})$, or $1 \%$ paraformaldehyde and $1 \%$ glutaraldehyde in $\mathrm{PB}$ ] overnight at $4^{\circ} \mathrm{C}$. The cord was then blocked, embedded in a 10\% gelatin matrix, postfixed, and cryoprotected in $20 \%$ sucrose overnight. Frozen transverse sections $(40-50 \mu \mathrm{m})$ were serially collected in $\mathrm{PB}$ and reacted with standard $\mathrm{ABC}-\mathrm{DAB}$ techniques (Elite kit; Vector Laboratories) to visualize neurobiotin. Sections were rinsed, mounted on slides, counterstained with neutral red, dehydrated, cleared, and coverslipped with Permount (Fisher Scientific).

To be included in the present analyses, multiple darkly stained central collaterals were required for each afferent. Labeled fibers were serially reconstructed at $200-1000 \times$ and drawn with the aid of a camera lucida. Photomicrographs of individual arborizations were obtained using a Dage (Dage-MTI, Michigan City, IN) 3CCD video camera with a CG-7 capture card (Scion, Frederick, MD). Image manipulation in Adobe Photoshop (Adobe Systems, San Jose, CA) routinely included minor adjustments of brightness and contrast to compensate for variable lighting at the time of image capture. Moreover, because of the limited depth of field at the higher magnifications needed to visualize the fine processes of neonatal afferents, multiple images were captured at different focal planes through individual sections and successively merged to generate a manually "Z-projected," in-focus stack as described previously (Woodbury et al., 2001). All photomicrographs in the present report, except that in Figure 5, represent such composite photomicrographs.

\section{Results}

The present report is based on observations on the physiology and central anatomy of 28 well labeled incipient myelinated skin sensory neurons from neonatal mice aged P1-P7. These 28 afferents represent a restricted subset of a much larger sample of well stained and characterized afferents ( 81 cells from 54 preparations aged P0-P7) spanning a diverse spectrum of skin sensory neurons from $\mathrm{C}$-fibers with broad, inflected somal action potentials to multiple subclasses of LTMRs with narrow, uninflected somal spikes; some of these were documented in a previous report (Woodbury et al., 2001). Importantly, all of the afferents in- 
Table 1. Physiological properties of incipient myelinated cutaneous HTMRs in neonatal mice

\begin{tabular}{|c|c|c|c|c|c|c|}
\hline & \multicolumn{3}{|c|}{ HTMRs (Postnatal age, $4.2 \pm 1.6 \mathrm{~d}$ ) } & \multicolumn{3}{|c|}{ LTMRs* (Postnatal age, $4.2 \pm 1.6 \mathrm{~d}$ ) } \\
\hline & All & Lamina I/IIo & Lamina I-V & All & RA & SA \\
\hline Mechanical threshold (mN) & $2.1 \pm 2.0(27)$ & $3.2 \pm 2.6(9)$ & $1.5 \pm 1.4(18)$ & $0.5 \pm 1.2(26)$ & $0.6 \pm 1.3(19)$ & $0.2 \pm 0.4(7)$ \\
\hline median & 2.45 & 2.45 & 0.87 & 0.07 & 0.07 & 0.07 \\
\hline Spike half-width (msec) & $2.1 \pm 0.7(26)$ & $2.6 \pm 0.7(8)$ & $1.9 \pm 0.5(18)$ & $0.8 \pm 0.1(18)$ & $0.8 \pm 0.1(12)$ & $0.8 \pm 0.1(6)$ \\
\hline median & 2.15 & 2.45 & 1.75 & 0.80 & 0.90 & 0.80 \\
\hline Spike amplitude (mV) & $70.7 \pm 12.3(26)$ & $69.5 \pm 15.6(8)$ & $70.7 \pm 11.1(18)$ & $52.4 \pm 12.5(18)$ & $55.8 \pm 11.6(12)$ & $45.6 \pm 12.4(6)$ \\
\hline median & 70.38 & 70.50 & 70.25 & 54.25 & 57.06 & 51.63 \\
\hline AHP half-width (msec) & $8.0 \pm 4.0(23)$ & $9.7 \pm 2.8(5)$ & $7.5 \pm 4.2(18)$ & $3.3 \pm 1.1(16)$ & $3.7 \pm 1.0(12)$ & $2.1 \pm 0.4(4)$ \\
\hline median & 7.00 & 9.30 & 6.40 & 3.35 & 3.80 & 2.15 \\
\hline Conduction velocity (m/sec) & $0.6 \pm 0.1(28)$ & $0.6 \pm 0.1(10)$ & $0.6 \pm 0.1(18)$ & $0.8 \pm 0.2(32)$ & $0.8 \pm 0.2(23)$ & $0.8 \pm 0.2(9)$ \\
\hline median & 0.57 & 0.53 & 0.61 & 0.73 & 0.71 & 0.81 \\
\hline
\end{tabular}

Values presented are mean \pm SD. Numbers of afferents included in each measurement are provided in parentheses.

*LTMR data are from afferents in Woodbury et al. (2001) and our unpublished observations.

cluded here displayed a diverse suite of key properties of adult myelinated HTMRs, sharing with the latter notable similarities in somal spike properties, mechanical thresholds, adaptation rate, increased sensitivity to increasing forces, receptive field organization, and central anatomy (see below). Because LTMRs are already well differentiated in terms of both physiology and anatomy at these same ages (Woodbury et al., 2001) (Table 1), the most parsimonious hypothesis is that this collection of neonatal afferents sharing multiple properties in common with adult myelinated nociceptors were indeed incipient myelinated HTMRs, and they are referred to as such in the following treatment.

\section{Myelinated HTMRs versus LTMRs in neonates}

Table 1 presents physiological data for all well stained incipient myelinated HTMRs from these studies for which spike data were available; these are compared with similar data from LTMRs across the same ages, some of which have been detailed previously (Woodbury et al., 2001). All afferents identified as HTMRs in the present study exhibited inflected somal spikes, and these were significantly greater in amplitude and duration [both spike and afterhyperpolarization (AHP) duration, measured at halfamplitude] than the uninflected spikes of LTMRs across the same ages $(p<0.0001$ for all three comparisons; Mann-Whitney $U$ test); indeed, there was no overlap in spike half-widths and only minimal overlap in AHP half-width measurements between these populations. In terms of peripheral physiology, all of these HTMRs exhibited SA response properties, and all but a distinctive subset (below) gave an increasingly vigorous response to increasing stimulus intensities; in contrast, the maximal response of LTMRs to higher forces could be reproduced with much lower forces. Overall, the mechanical thresholds of these afferents were significantly higher than those of LTMRs $(p<0.0001$; MannWhitney $U$ test). Importantly, the values in Table 1 are artifactually inflated, because the thresholds of the majority of neonatal LTMRs (18 of 26) were below the limit of resolution $(0.07 \mathrm{mN})$ of von Frey filaments. Nevertheless, the remaining LTMRs exhibited thresholds equal to or above the lowest value seen among HTMRs $(0.3 \mathrm{mN})$. Thus, mechanical threshold in isolation could not reliably separate these neonatal populations a priori; somal spike and physiological response properties were found to be far more robust predictors of afferent modality.

Across this sample of neonatal HTMRs, there was considerable variation in conduction velocity, spike width, mechanical threshold, and rate of adaptation to sustained stimuli, as has been noted in adult HTMRs (Burgess and Perl, 1967; Koerber et al., 1988). On the basis of central anatomy, two subpopulations could be distinguished (Table 1), one projecting to laminas I and
IIo, the other arborizing throughout all DH laminas. In view of this striking discontinuity, afferents were subdivided further on morphological grounds to examine the range of physiological diversity within these subpopulations.

\section{Lamina I/IIo HTMRs}

As seen in Figures 1 and 2, the central projections of this population were centered primarily on the marginal zone. In all, 10 well labeled examples of this class have been recovered from neonates across ages $\mathrm{P} 1(n=1), \mathrm{P} 2(n=1), \mathrm{P} 3(n=1), \mathrm{P} 4(n=2), \mathrm{P} 6(n=$ $2)$, and P7 $(n=3)$; these were similar overall to another, albeit less well labeled, afferent from a P3 neonate. Across incipient myelinated afferents, their peripheral CVs were relatively slow (Table 1; range, $0.43-0.77 \mathrm{~m} / \mathrm{sec}$ ); among all afferents in any given nerve, their latencies to electrical stimulation were generally intermediate overall, typically twice that of the fastest afferents and at least one-half that of future unmyelinated afferents. Unlike LTMRs across the same ages, all were characterized by broad somal action potentials with an inflection on the falling phase, representative examples of which are illustrated in Figures 1 and 2.

\section{Peripheral physiology}

RFs of these afferents were typically small $\left(\sim 1 \mathrm{~mm}^{2}\right)$ and spotlike; occasionally, multiple sensitive foci separated by regions of lower sensitivity could be discerned. Included in this population were afferents exhibiting the highest mechanical thresholds seen among neonatal afferents (Table 1; range, 0.3-7.8 mN), significantly above those of different populations of LTMRs across the same ages ( $p<0.001$; Mann-Whitney $U$ test).

Despite their generally elevated mechanical thresholds, these afferents were very responsive to skin stimulation when first encountered. For example, gentle probing with a blunt glass stylus was sufficient to locate the RFs of all but one afferent (Fig. 2B), whose RF could not be found (see caveats by Woodbury et al., 2001). Although SA responses could normally be elicited in these afferents using suprathreshold von Frey filaments, the typical response to the latter was a burst of spikes that tended to adapt out fairly rapidly to maintained stimuli. Furthermore, maximal responses were often obtained at relatively low von Frey forces (Fig. 1), such that a number of these afferents failed to respond to increasing stimulus intensities with incrementally graded activity. Rather, they tended to habituate and/or desensitize to repetitive stimulation, with some rendered completely inexcitable by an intense stimulus. In a few cases, activity could again be elicited by stimulation at a different spot. It should be noted that these afferents invariably responded far more vigorously to a sharp 
object (e.g., number 5 forceps) than to blunt von Frey filaments, although the former were generally avoided in view of the above physiological consequences and lack of force control.

Interestingly, repetitive stimulation gave rise to a pronounced, long-lasting hyperpolarization in many of these afferents. This is evident in both the fast and slow sweep speeds in Figure 1. As seen there, even relatively low-frequency (i.e., $0.5 \mathrm{~Hz}$ ) electrical nerve stimuli led to a progressive hyperpolarization (Fig. 1,E), although this membrane effect was greatest when spikes followed in rapid succession (e.g., during natural stimulation) (Fig. 1, $G S, v F)$. Although a similar membrane effect was also seen in other HTMRs (see Fig. 4), it was most pronounced in this subpopulation; its potential functional significance and underlying cause(s) will be discussed elsewhere.

\section{Central anatomy}

As noted above, the central projections of these afferents were centered primarily on laminas I and IIo and thus closely approximated the morphology of a distinctive subset of adult thinly myelinated HTMRs (Light and Perl, 1979). The range of central anatomic variation seen among neonatal afferents of this type, the earliest from a $\mathrm{P} 1$ neonate, is illustrated by the examples shown in Figures 1 and 2. On entry into the cord, their axons bifurcated to give rise to ascending and descending axons that could normally be traced over multiple segments. A small number of these afferents gave rise to axons ascending in the dorsal column (Fig. 2B); however, the majority remained lateral after entry into the cord, ascending in the vicinity of Lissauer's tract or even farther laterally in the thin veil of white matter overlying the dorsolateral edge of the $\mathrm{DH}$.

Regardless of the location of their ascending-descending axons, all of these afferents arborized in a relatively tight plexus conforming to the outline of lamina I. Altogether, they gave rise to extremely fine central processes that exhibited a complex branching pattern in the cord. The greatest density of collateral arbors was seen nearest the dorsal root entry zone, and, as illustrated in Figure 1, this primary projection was located in somatotopically appropriate locations across the mediolateral axis of the DH. The RF of the afferent to the left in Figure 1 was located near the dorsal midline, and its central arbors were located at the lateral edge of the marginal zone; to reach this lateral position, collaterals followed long curving arcs through the transverse plane of lamina I in a manner mirroring the classic central anatomy of these afferents (Light and Perl, 1979). In contrast, the RF of the afferent to the right in Figure 1 was near the ventral edge
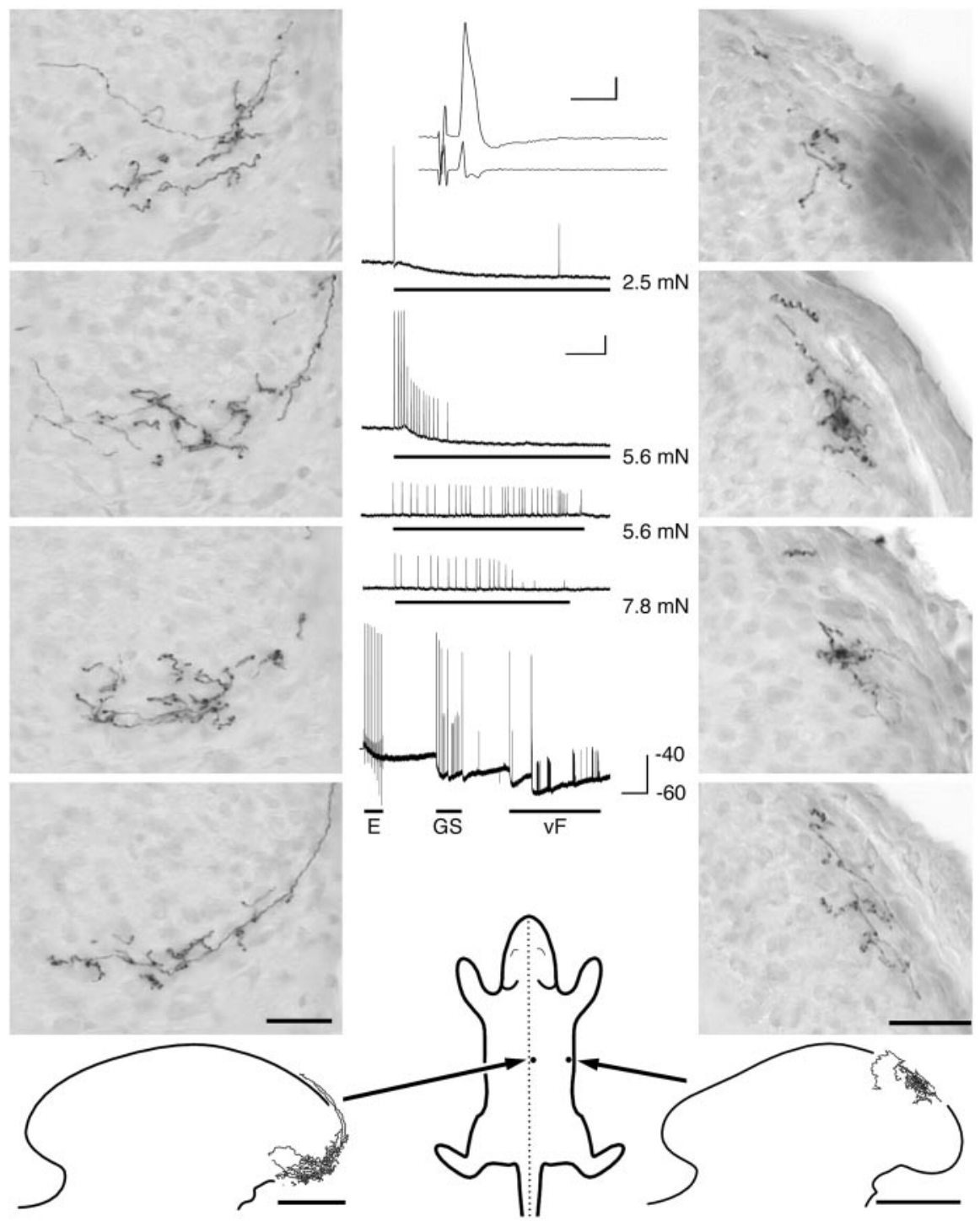

Figure 1. Central anatomy, physiology, and somatotopic organization of lamina I//lo HTMRs in neonates. Photomicrographs of serial sections through the central arbors of two different HTMRs from neonates aged P6 (left) and P3 (right). Note that, although arborizations are centered primarily on lamina I, processes also extended into deeper laminas. Camera lucida reconstructions (bottom) and RF locations (mouse pup schematic) illustrate that the projections of these afferents are somatotopically appropriate. Physiological records (center, obtained from the afferent at left) illustrate the broad inflected somal action potential and evoked activity to graded forces (durations indicated by solid bars). Note that the response adapted rapidly in many cases and became saturated at relatively low stimulus intensities. Note also the pronounced activity-induced hyperpolarization. Bottom trace shows the entire recording of this neuron, in which this hyperpolarization is readily apparent. E, Electrical nerve stimuli; GS, gentle probing with blunt glass stylus; $v F$, testing with incrementally graded von Frey filaments. Thresholds and CVs, respectively, were $2.5 \mathrm{mN}$ and $0.56 \mathrm{~m} / \mathrm{sec}$ (left) and $0.3 \mathrm{mN}$ and $0.53 \mathrm{~m} / \mathrm{sec}$ (right). Scale bars: photomicrographs, $25 \mu \mathrm{m}$; camera lucida reconstructions, 100 $\mu \mathrm{m}$. Calibration: $10 \mathrm{mV}, 5 \mathrm{msec}$ (spike), $200 \mathrm{msec}$ (naturally evoked responses), and $20 \mathrm{sec}$ (complete trace).

of the DCN innervation territory, and its projections were located medially within the projection zone of the DCN. In large part, therefore, this population of afferents exhibited a somatotopic organization similar to that seen among LTMRs (Woodbury et al., 2001). In contrast to the latter, however, the somatotopy in the mediolateral axis among these afferents appeared relatively coarse, because many gave rise to one or more collaterals that arborized in a mediolateral location outside the primary projection (Fig. 2).

Although the bulk of the arborization was centered on lamina I, all gave rise to a diffuse meshwork of processes deep to the marginal layer, the extent of which varied not only between affer- 

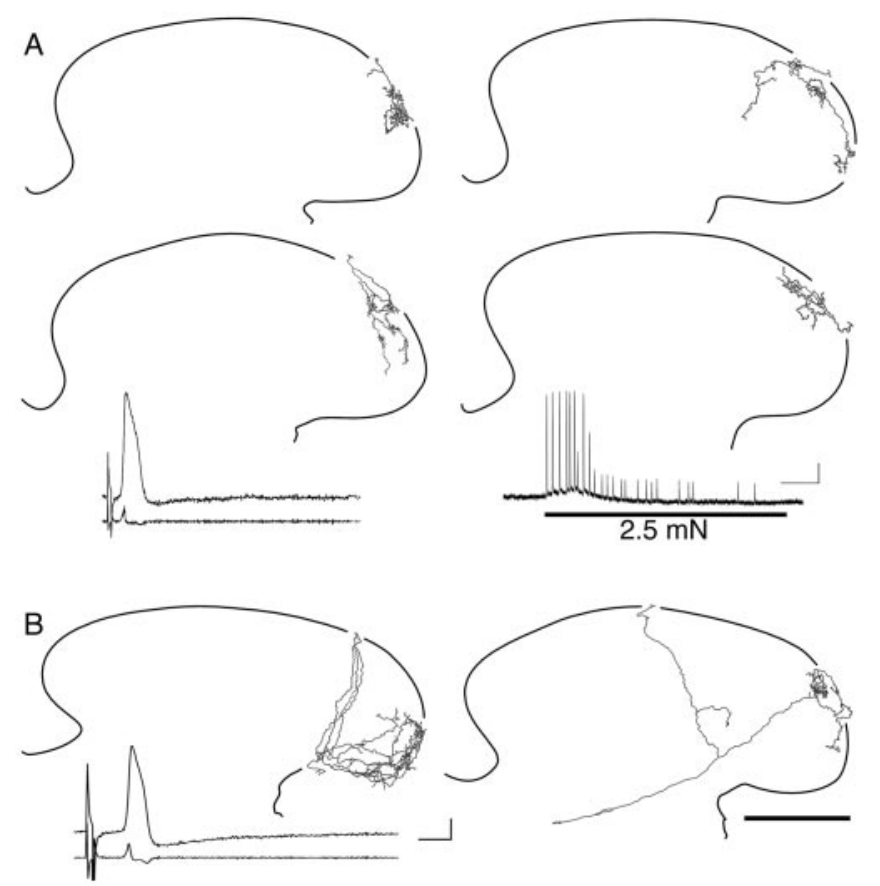

Figure 2. Diversity of central collateral morphology among lamina I/llo HTMRs. Camera lucida reconstructions of the collateral arbors from HTMRs in neonates aged P1 $(A)$ and P2 (B). Note that, although these collaterals were oriented primarily in the horizontal plane parallel to the marginal zone, they also extend to variable depths into deeper laminas. The central axons of most lamina I/Ilo HTMRs remained laterally as in $A$, although some coursed in the dorsal column as shown in $B$. Spike traces and derivatives reveal the broad inflected somal action potentials typical for these afferents. Naturally evoked $S A$ response to a sustained stimulus $(2.5 \mathrm{mN}$, duration indicated by the solid bar). CVs were $0.43 \mathrm{~m} / \mathrm{sec}(A)$ and $0.40 \mathrm{~m} / \mathrm{sec}(B)$. Threshold for $A$ was $0.7 \mathrm{mN}$; RF for $B$ could not be located, although another lamina I/llo HTMR with identical morphology and somal spike had a threshold of $2.5 \mathrm{mN}$ and response properties similar to those in $A$. Scale bars, $100 \mu \mathrm{m}$. Calibration: $20 \mathrm{mV}, 5 \mathrm{msec}$ (spike), $250 \mathrm{msec}$ (naturally evoked response).

ents but also between collaterals from a single afferent. All projected extensively into the outer half of the incipient substantia gelatinosa (i.e., lamina IIo), in which they would clearly overlap with unmyelinated inputs. The majority also gave rise to small numbers of processes coursing throughout deeper regions of the DH occupied by the collateral arbors of low-threshold afferents (Figs. 1, 2). Hence, the central projections of myelinated HTMRs invade regions occupied by both unmyelinated and tactile afferents in early postnatal life; as seen below, this was also the case for other myelinated afferents encoding stimulus intensity.

\section{Lamina I-V HTMRs}

Over the course of early experiments aimed at sampling the broad spectrum of skin sensory neurons in neonates, incipient myelinated HTMRs exhibiting a distinctly novel central morphology were encountered, and later experiments attempted to target this group selectively. In all, a total of 18 well labeled and characterized examples have been recovered from neonates across ages P2 $(n=1), \mathrm{P} 3(n=4), \mathrm{P} 4(n=9), \mathrm{P} 5(n=2), \mathrm{P} 6(n=1)$, and P7 $(n=1)$; these were similar anatomically to two additional well stained afferents from a P3 and a P5 neonate for which RFs could not be found.

Overall, this sample was characterized by a broad range of CVs $(0.35-0.97 \mathrm{~m} / \mathrm{sec})$ (Table 1$)$. Although only slightly above lamina I/IIo HTMRs on average ( $p=0.2$; Mann-Whitney $U$ test), the CVs of some overlapped with future large-diameter (A $\beta)$ LTMRs at these same ages (e.g., guard hair and touch dome afferents). As with lamina I/IIo HTMRs, all exhibited relatively largeamplitude $(>50 \mathrm{mV})$, inflected somal spikes, although greater variation in spike and AHP duration was seen in this group. A small number exhibited broad spikes that were indistinguishable from those of lamina I/IIo HTMRs. On average, however, these spikes were significantly narrower (range, $1.1-3.0 \mathrm{msec}$ ) than those of lamina I/IIo HTMRs (range, 2.1-4.2 msec; $p=0.02$; Mann-Whitney $U$ test) but were nevertheless significantly broader than LTMRs at the same ages (Table 1$)(p<0.001$; Mann-Whitney $U$ test). In the narrowest spikes (also among the fastest conducting), only a slight inflection could be discerned. On close inspection, however, an inflection marked by an $\sim 45^{\circ}$ break in the derivative was apparent throughout. Importantly, even the narrowest spikes among these did not overlap in spike width with the broadest LTMR spikes; an example comparing the two from the same animal is illustrated in Figure 5 (see also Fig. 6). Interestingly, nearly identical narrow spikes have been observed in A $\beta$ HTMRs from adult mice (data not shown), which are clearly narrower than the spikes of A $\delta$ HTMRs (Koerber et al., 1988).

Peripheral physiology

As with spike duration, this group of afferents constituted an intermediate population in terms of mechanical threshold. Although all were equal to or above the minimum threshold observed among lamina I/IIo HTMRs, thresholds tended to be lower in this group (Table 1) (range, $0.3-5.6 \mathrm{mN} ; p=0.09$; Mann-Whitney $U$ test). Nevertheless, thresholds were significantly higher overall than those of LTMRs across these same ages (Table 1) ( $p<0.001$; Mann-Whitney $U$ test).

Unlike many lamina I/IIo HTMRs (above), all of these afferents responded with increasing discharge to increasing forces. However, there were notable differences in sensitivity within this group, and two basic types of response were observed overall, as illustrated in Figures 3 and 4. Many gave rise to a sluggish, fairly rapidly adapting (RA) response typical of lamina I/IIo HTMRs (Fig. 3). In contrast, others were relatively sensitive and more slowly adapting (Fig. 4). Interestingly, a few among these responded phasically to stimulation over a large $\left(>2 \mathrm{~mm}^{2}\right)$, illdefined area. However, careful probing revealed a much smaller region of heightened sensitivity in which suprathreshold stimuli elicited a fairly regular response that became increasingly vigorous at higher forces (Fig. 4). The relatively low thresholds and increasingly vigorous responses at higher forces imparted a wide dynamic range to many of these afferents. None exhibited resting background activity despite the skin being stretched slightly and pinned flat, and none specifically tested gave any response to dorsoventral or rostrocaudal skin stretch. As with lamina I/IIo HTMRs, multiple spots of heightened sensitivity could be discerned on occasion within the RFs of these more sensitive HTMRs. Interestingly, similar RF properties, including some with an ill-defined RA "surround," have been seen among $A \beta$ HTMRs recorded in an identical adult preparation (data not shown). These more sensitive afferents may correspond to the generally overlooked "moderate pressure" receptors identified in seminal studies of myelinated nociceptors (Burgess and Perl, 1967; Perl, 1968), many of which conduct in the $A \beta$ range (Burgess and Perl, 1967; Koerber et al., 1988).

\section{Central anatomy}

In contrast to the majority of lamina I/IIo HTMRs whose longitudinal axons remained laterally (above), the central axons from all of these afferents ascended and descended in the dorsal col- 

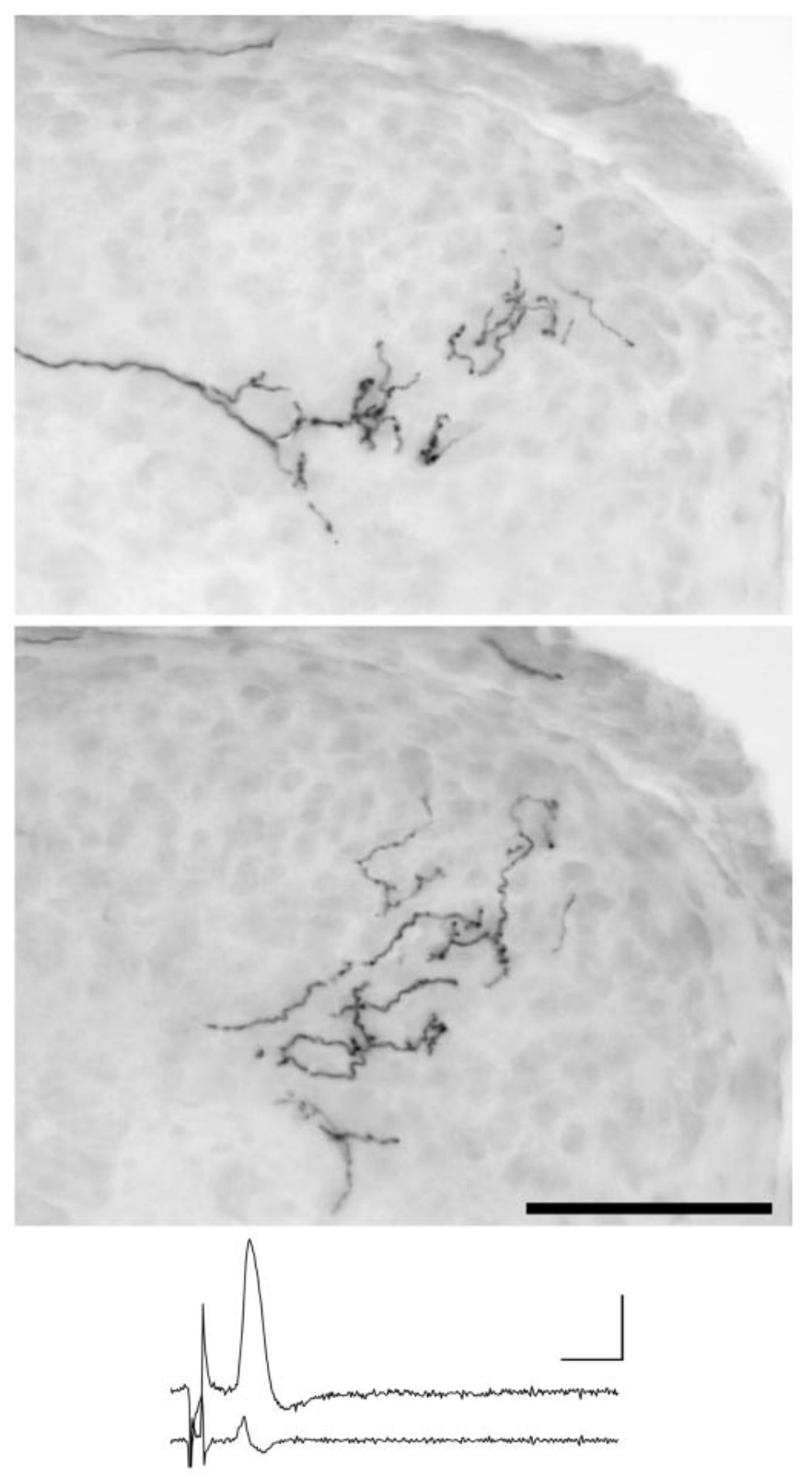

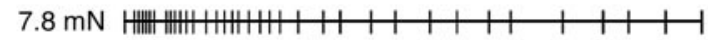
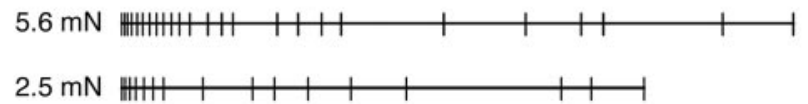

$0.74 \mathrm{mN} \longmapsto+1+1+1+1+1+14$

Figure 3. Serial photomicrographs of a collateral arborization from a lamina I-V HTMR from a P5 neonate. Note the dorsally recurving flame-shaped appearance of this arbor, which extended diffusely throughout all laminas into the marginal zone. Although the spike was narrower than those of lamina I/Ilo HTMRs (compare Figs. 1, 2), the peripheral response properties of this afferent were nearly indistinguishable, with the exception that these afferents were better able to encode stimulus intensity. Threshold and $\mathrm{CV}$ were $0.3 \mathrm{mN}$ and $0.46 \mathrm{~m} / \mathrm{sec}$, respectively. Scale bar, $50 \mu \mathrm{m}$. Calibration: $20 \mathrm{mV}, 5 \mathrm{msec}$ (spike), $200 \mathrm{msec}$ (evoked response raster).

umn. As exemplified in Figures 3 and 4, considerable diversity in collateral morphology was seen among this group. Collateral processes ranged in size from extremely fine to relatively coarse. Collaterals curved laterally after penetrating the gray matter to arborize within somatotopically appropriate areas; many were reminiscent of the dorsally recurving "flame-shaped" arbors of- ten identified with hair follicle afferents (compare Figs. 3, 5). In marked contrast to LTMRs, however, the arbors of this group extended throughout both deep and superficial DH regions, including the outer substantia gelatinosa and marginal zone. Although a small number of these afferents gave rise to relatively minor projections to lamina I, most gave rise to large numbers of processes in this outer later. On reaching the latter, processes typically altered their trajectory to continue horizontally along either transverse or rostrocaudal axes while arborizing further (Fig. 4). Moreover, they formed a characteristically diffuse, reticular meshwork of processes extending evenly throughout all laminas. Most were relatively expansive across the mediolateral axis; in one, a single collateral branched repeatedly across deeper $\mathrm{DH}$ to give rise to three or four mediolaterally separate flame-shaped arbors, as noted in Golgi studies (Scheibel and Scheibel, 1968). Extremely fine en passant varicosities were scattered along processes as they passed throughout all laminas. The characteristically diffuse nature of these arbors, in contrast to a typical LTMR arbor, is exemplified in Figure 5.

Despite the morphological diversity among these arbors, no consistent physiological correlates could be detected. Thus, afferents exhibiting broad spikes and a sluggish, fairly rapidly adapting response in the manner of lamina I/IIo HTMRs could be either flame-shaped with relatively minor projections into lamina I or far more mediolaterally expansive with large numbers of processes in lamina I. Similar diversity was also evident across the more sensitive, slowly adapting group. Indeed, the collaterals of some afferents displayed both extremes, and thus this apparent diversity may represent a morphological continuum.

\section{Postnatal fate}

In light of the results of cholera toxin bulk-transport studies (Fitzgerald et al., 1994), it might be predicted that these distinctive myelinated HTMR arborizations throughout the entire DH represent a transient developmental phenotype, particularly because adult bulk-labeling patterns showing a lack of myelinated inputs in superficial pain-specific dorsal horn laminas (I/IIo) are achieved by the end of the second postnatal week (but see Woodbury et al., 2000). As seen in Figure 6, however, preliminary studies reveal that the arbors of these afferents are still present in superficial DH laminas at 3 weeks of age. This afferent displayed no obvious signs of regression (e.g., retraction bulbs) and indeed supported multiple collaterals with robust arbors similar to the two different ones represented in Figure 6, $A$ and $B$. This raises the intriguing possibility that these extensive projections remain into adulthood, although they may not be obvious in bulk-labeling studies.

Figure $6 C-F$ also reveals that the narrow somal spikes of these sensitive HTMRs seem to show little change in width postnatally. In Figure 6E, this somal spike from a 3-week-old mouse is shown superimposed on the spikes from two other HTMRs with essentially identical peripheral response properties and mechanical thresholds, one recorded from a P2 neonate and the other at P8. Importantly, these two neonatal spikes were the narrowest among the entire sample of neonatal HTMRs yet are essentially identical to the spike from this 3-week-old mouse. Conversely, even after 3 weeks, this narrow HTMR spike is still broader than that from a P1 LTMR (Fig. 6F). Although certain alterations in somal spike properties are likely to occur postnatally (Fitzgerald and Fulton, 1992), the present findings suggest that, to understand these changes fully, it will be important to confine comparisons within discrete subsets of identified afferents. 


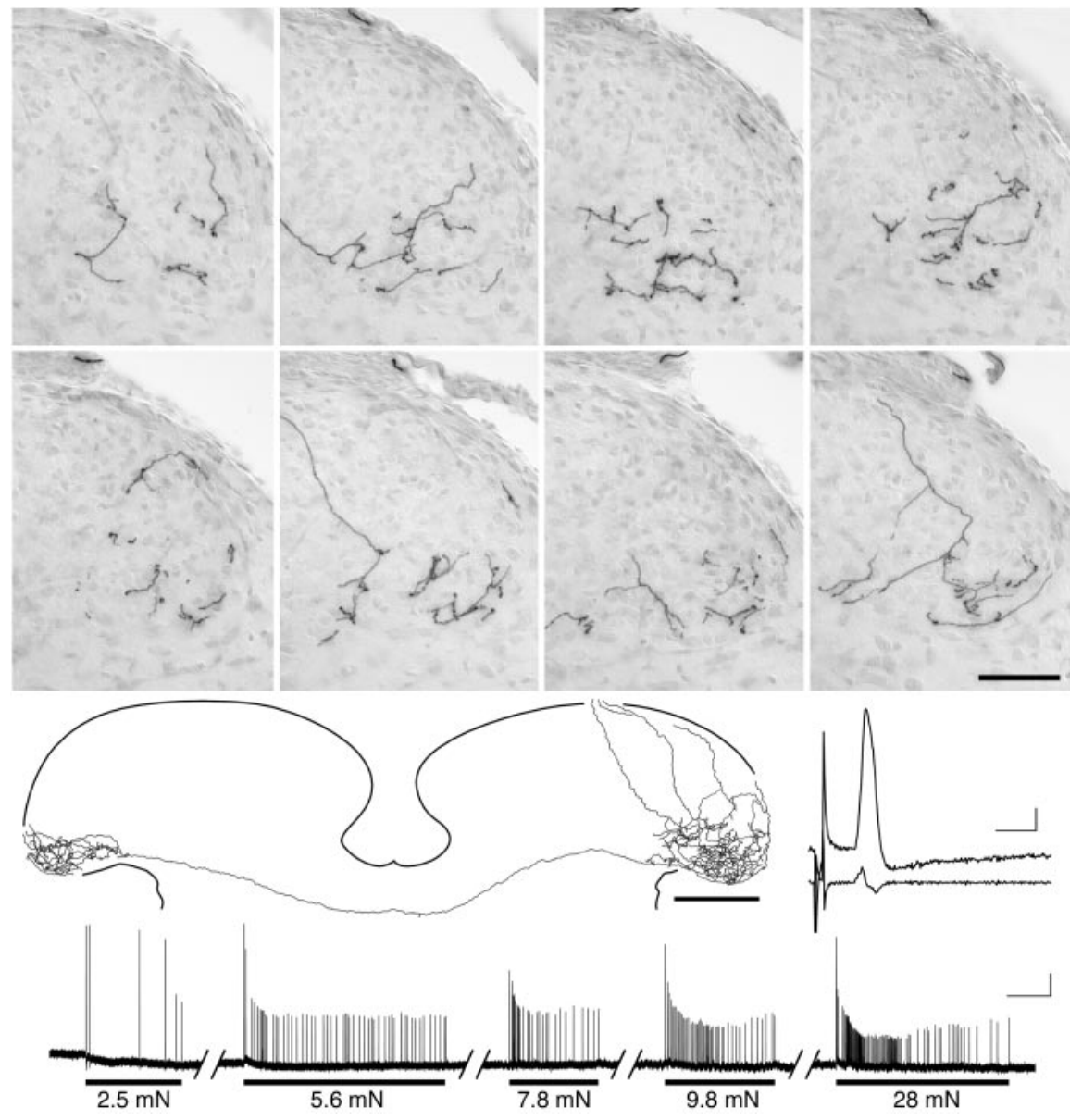

Figure 4. Central projections of a lamina I-V HTMR from a P6 neonate. Photomicrographs show serial sections through the arbors of three separate collaterals (rostrocaudal from top left to bottom right). Note the diffuse network of processes throughout the entire DH, exemplified in camera lucida reconstruction (center). Note also that the narrow, slightly inflected somal spike was nearly identical to that of the afferent shown in Figure 3. Evoked activity traces (bottom) show that the fairly regular response of these afferents to incrementally graded forces became increasingly vigorous at higher forces. Threshold and $\mathrm{CV}$ were $2.5 \mathrm{mN}$ and $0.66 \mathrm{~m} / \mathrm{sec}$. Scale bars: photomicrographs, $50 \mu \mathrm{m}$; camera lucida, $100 \mu \mathrm{m}$. Calibration: $10 \mathrm{mV}, 5 \mathrm{msec}$ (spike), $200 \mathrm{msec}$ (naturally evoked responses).

\section{Discussion}

The present report describes the central morphology of single, physiologically characterized, incipient myelinated HTMRs in neonatal mice. As with recent studies on neonatal LTMRs (Woodbury et al., 2001), these studies used an intact, ex vivo somatosensory system preparation to allow intrasomal physiological characterization and staining of individual cutaneous afferents. The resulting suite of diverse physiological and anatomic data allowed a comprehensive, "total-evidence" approach to the identification and analyses of individual developing neurons. Relative to LTMRs at these same ages, the afferents included here exhibited higher mechanical thresholds and responded to incrementally graded forces with an increasingly vigorous, slowly adapting discharge. They also exhibited relatively broad somal spikes with an inflection on the falling phase. These data, taken in combination, suggest that these afferents were indeed developing HTMRs. As in adults, these neonatal HTMRs exhibited considerable diversity across a variety of physiological parameters. Anatomically, two central phenotypes were evident, both of which projected into the substantia gelatinosa and marginal layer, in which they would overlap extensively with unmyelinated afferent inputs. In nearly all respects, these afferents, like neonatal LTMRs, were simply miniaturized versions of their adult counterparts. These findings shed new light on wide-ranging issues, from the acquisition of physiological identity to the development and functional organization of spinal pain centers.

\section{Adult-like physiological properties among sensory neurons in early postnatal life}

Somal spike shape

The present studies of myelinated HTMRs in neonatal mice, in conjunction with findings from diverse subpopulations of LTMRs over the same time period (Woodbury et al., 2001), represent the earliest intracellular recordings to date of somal action potentials from identified cutaneous primary afferents. Combined, these studies reveal that somal spikes are adult-like in all major respects shortly after birth (in contrast to Fulton, 1987; Fitzgerald and Fulton, 1992). That is, myelinated LTMRs exhibit narrow, uninflected somal spikes, myelinated HTMRs exhibit inflected spikes ranging from broad to relatively narrow, and unmyelinated (C) afferents exhibit broad, inflected somal spikes in newborns as in adults (Rose et al., 1986; Koerber et al., 1988; Ritter and Mendell, 1992; Djouhri et al., 1998).

The developmental time point at which these stereotypical somal spike properties are acquired is not known, although there is evidence to suggest that it occurs very early in embryonic life. In embryonic rat DRG neurons, for example, narrow (i.e., adult-like) uninflected spikes (Mirnics and Koerber, 1997) and adultlike expression patterns of TTX-sensitive and TTX-insensitive sodium currents (Ogata and Tatebayashi, 1992) are already in place by embryonic day 17 (the earliest time point studied). Moreover, in Xenopus tadpoles, in which the earliest in situ recordings from DRG neurons have been obtained, a remarkable diversity of somal action potentials, including large numbers of narrow uninflected TTX-sensitive spikes, is in evidence from the earliest time point onward (Baccaglini, 1978). The intriguing possibility that primary afferent physiology may be predetermined seems to have a molecular parallel (Rifkin et al., 2000) and clearly warrants additional investigation.

\section{Primary afferent diversity}

Along with adult-like somal spikes, these studies found that the skin of newborn mice is innervated by a diverse functional array of myelinated afferents, including multiple subclasses of LTMRs (Woodbury et al., 2001) and HTMRs (present study) that are in all major physiological respects identical to their adult counterparts. This finding is in accord, therefore, with the pioneering studies of Fitzgerald (1987) in neonatal rat and stands in contrast to an early report from the present laboratory (Ritter et al., 2000) in which a significant selection bias against LTMRs (Djouhri and 


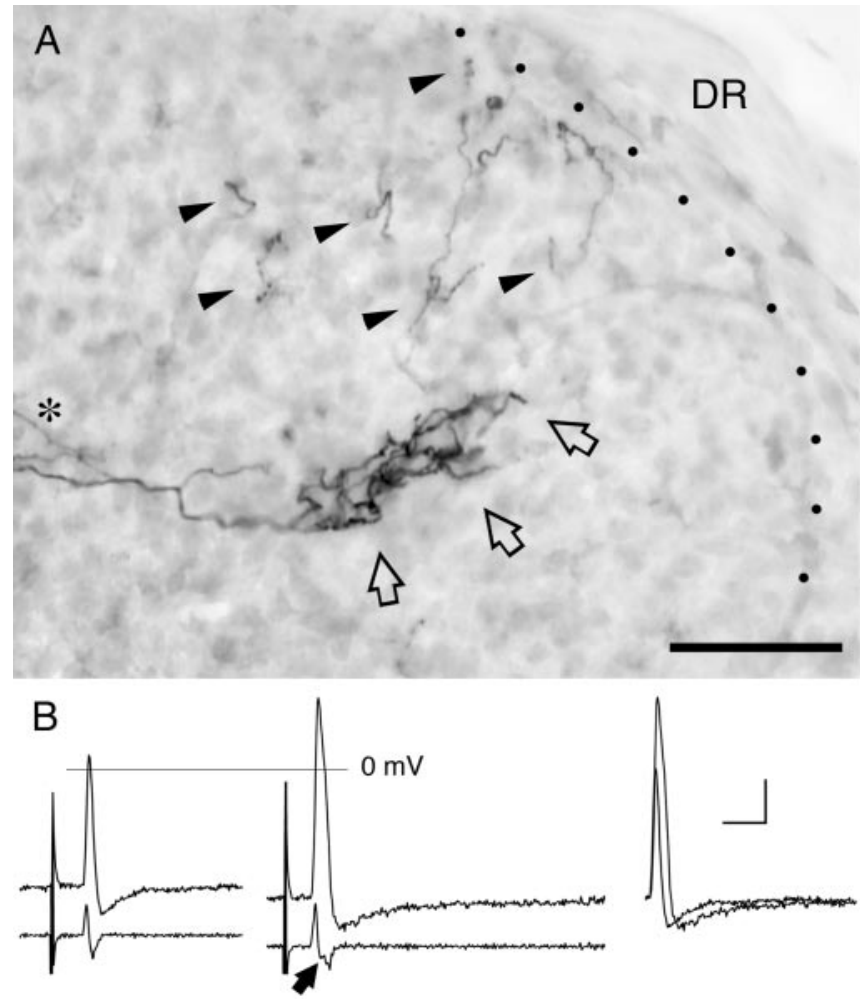

Figure 5. Differences between LTMRs and lamina I-V HTMRs in neonates. A, Photomicrograph of the DH arborizations of a hair follicle afferent (open arrows) and a myelinated HTMR (arrowheads) in a single section from a P5 neonate. Afferents were injected in adjacent DRGs and fully reconstructed to identify their central processes in regions of overlap; the location of the RF of the more laterally projecting LTMR was dorsal to that of the HTMR. Note the dense arborization of the LTMR and its depth from the gray-white border (dotted line) as noted previously (Woodbury et al., 2001). In contrast, the central arbors of the HTMR spread diffusely over a broader area throughout the entire $\mathrm{DH}$; its recurving collateral axons (asterisk) can be seen paralleling the larger axon of the LTMR. B, Somal action potential traces and derivatives from these same afferents. Note that, relative to the somal spike of the LTMR (left), the HTMR spike has a distinct inflection on the falling limb (arrow in derivative trace); it is also greater in amplitude and duration, readily apparent when these spikes are superimposed (right). Mechanical thresholds were $<0.07$ and $2.5 \mathrm{mN}$ for the LTMR and HTMR, respectively; the LTMR was typically rapidly adapting, whereas the HTMR gave a slowly adapting response to forces $>5$ $\mathrm{mN}$. DR, Dorsal root. Scale bar, $50 \mu \mathrm{m}$. Calibration: $20 \mathrm{mV}, 5 \mathrm{msec}$.

Lawson, 2001; Woodbury et al., 2001) was compounded by the small sample size. Interestingly, most anatomically verified HTMRs in the present study were overtly similar in mechanical thresholds and response properties to units that composed a transient "pressure" category in earlier extracellular studies (Fitzgerald, 1987). For example, the physiological properties ascribed to "RA pressure" units were commonly observed among lamina I/IIo HTMRs, whereas those ascribed to "SA pressure" units were common among lamina I-V HTMRs (HTMRs in this earlier study, in contrast, may have represented predominantly unmyelinated HTMRs) (Fitzgerald, 1987, her Fig. 1). It is noteworthy, however, that considerable physiological overlap was seen between these two anatomic subpopulations. For example, many lamina I-V HTMRs displayed somal spikes and peripheral response properties that were outwardly identical to those seen among lamina I/IIo HTMRs; hence, these two populations could not be reliably differentiated a priori with the manual techniques used. It is possible that distinct physiological differences may be revealed between these neonatal populations through the application of true (i.e., feedback-controlled) constant-force stimuli. Al-

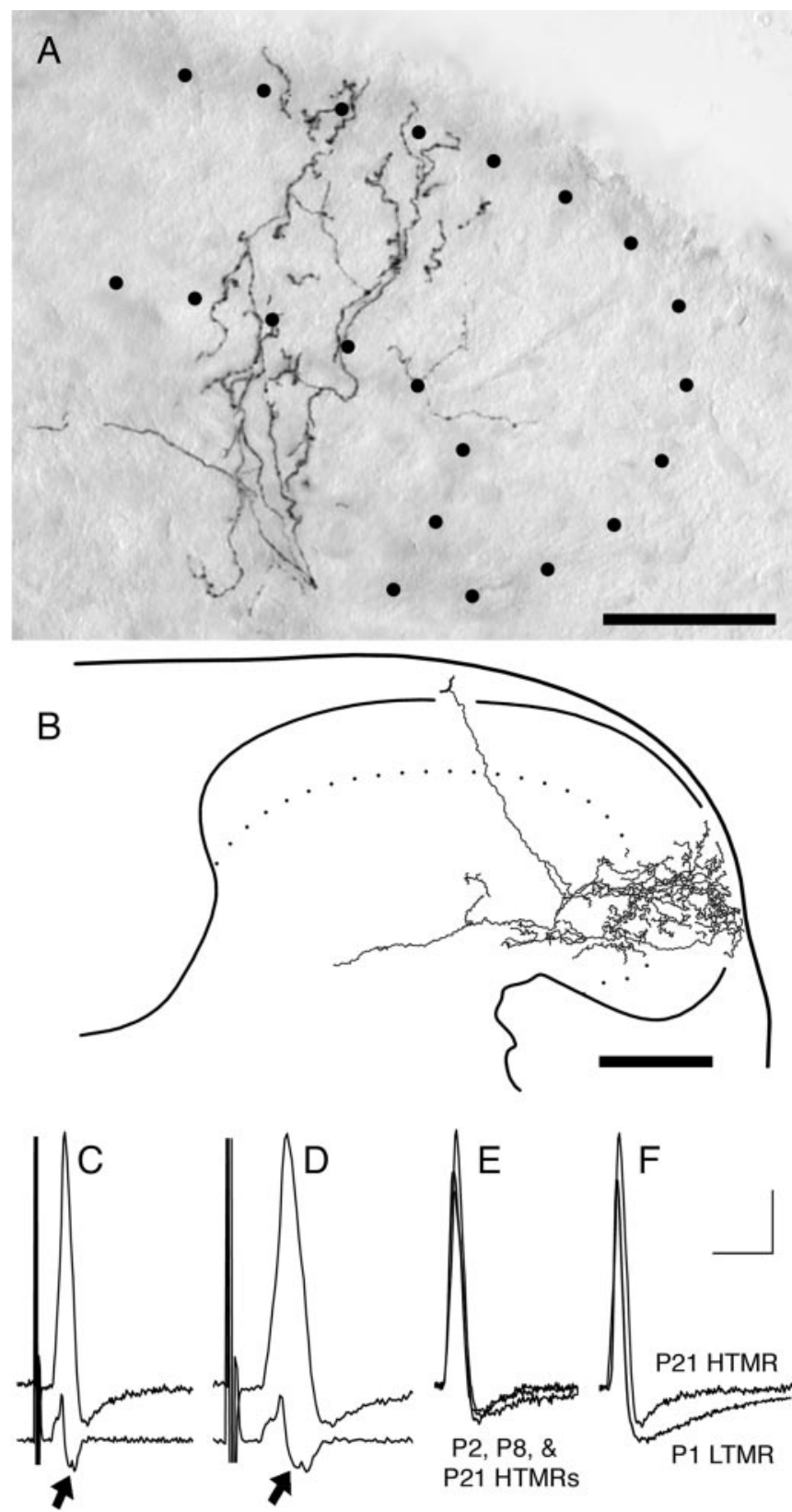

Figure 6. Central projections of a lamina I-V HTMR from a 3-week-old mouse. A, Photomicrograph of the arborization in a single section; dotted lines indicate boundaries of laminas I/II. $B$, Camera lucida reconstruction of a different collateral arbor. C, Narrow, slightly inflected spike of this afferent, shown at expanded scale in D; arrows mark the break in the derivative. E, The spike from this P21 afferent is shown superimposed on spikes from two other sensitive lamina I-VHTMRs, recorded in P2 and P8 neonates; note the close match in spike and AHP width. F, This same P21 spike is shown superimposed on the even narrower spike of an LTMR recorded in a P1 neonate. Scale bar, $50 \mu \mathrm{m}$. Calibration: $20 \mathrm{mV}, 5 \mathrm{msec}$ (except $D$, which is $2.5 \mathrm{msec}$ ).

ternatively, physiological differences may emerge at later time points, possibly via a shift toward higher mechanical thresholds among certain subsets. Although the range of mechanical thresholds across these neonatal HTMRs was remarkably similar to that observed to date across HTMRs (both $\mathrm{A} \delta$ and $\mathrm{A} \beta$ ) in an identical adult preparation (data not shown), not all of the latter have been identified centrally; thus, future comparisons confined within anatomically defined subpopulations will be necessary to elucidate the ontogeny of mechanical thresholds among HTMRs. 


\section{Myelinated HTMRs project throughout the substantia gelatinosa}

The present finding of two basic morphological phenotypes among cutaneous myelinated HTMRs in neonatal mice confirms initial studies of the spinal projections of these afferents in adult cats and primates (Light and Perl, 1979; Rethelyi et al., 1982; Traub and Mendell, 1988). One neonatal population, exhibiting the highest mechanical thresholds, mirrored the central morphology of a distinctive subset of adult thinly myelinated (A $\delta$ ) HTMRs that contribute to Lissaur's tract and terminate in the marginal zone, noted in studies of both cutaneous (Light and Perl, 1979; Hayashi, 1985) and deep tissue nociceptors (Hoheisel et al., 1989). These may correspond to the "specific nociceptors" identified in early studies (Burgess and Perl, 1967; Perl, 1968). However, in addition to a predominant projection to lamina I and additional inputs to IIo as noted in adults, these neonatal lamina I/IIo HTMRs also sent sparse projections into deeper laminas. The other population differed dramatically from this classic phenotype, with axons in the dorsal columns and recurrent, often flame-shaped collateral arbors forming a diffuse, reticular meshwork throughout all DH laminas. This latter population was physiologically diverse and may therefore encompass the two other subcategories of HTMRs identified originally (lowsensitivity mechanoreceptors and moderate-pressure afferents) (Burgess and Perl, 1967; Perl, 1968).

The extent to which these widespread central projections from neonatal HTMRs represent a transitory "exuberance" is not clear at present. Part of this uncertainty stems from possible species variability. For example, HTMRs are more extensive centrally in monkeys than in cats, projecting throughout lamina IV as well as laminas I and V (Rethelyi et al., 1982). That the full spectrum of anatomic diversity among HTMRs is not yet adequately understood is also reflected in the fact that cutaneous moderatepressure units (Burgess and Perl, 1967) have not yet been characterized centrally in adults. Nevertheless, preliminary findings that these extensive projections still remain in 3-week-old mice (Fig. 6), at a time when bulk-transport studies indicate otherwise (Fitzgerald et al., 1994; Woodbury et al., 2000), suggest that such inputs from myelinated afferents to the substantia gelatinosa may remain throughout life. Their characteristically diffuse nature, particularly when contrasted against the dense arbors of LTMRs (Fig. 5), may account for the apparent inability of bulk-labeling methods to reveal these inputs in adults under normal conditions (Woolf et al., 1992) (but see Tong et al., 1999; Woodbury et al., 2000 for nonrodents). It is important to note that, although this unique fibroarchitecture may persist, reorganization of myelinated inputs in the substantia gelatinosa appears to continue well beyond 3 weeks of age (Park et al., 1999; Nakatsuka et al., 2000), and thus the synaptic efficacy and/or connectivity of these inputs may be quite plastic.

\section{Neonatal pain}

These findings, in conjunction with recent studies of neonatal LTMRs (Woodbury et al., 2001), shed important new light on the development of spinal pathways underlying nocifensive behaviors. They reveal that inputs into nocireceptive $\mathrm{DH}$ regions from myelinated afferents in neonates arise from afferents that encode stimulus intensity (i.e., nociceptors) and not LTMRs. It is well documented that protective withdrawal reflexes exhibit marked hypersensitivity in neonatal mammals (for review, see Fitzgerald and Jennings, 1999), with adult-like withdrawal reflex patterns attained at $\sim 3$ weeks of age (Holmberg and Schouenborg, 1996). Interestingly, activation of these reflexes in adults requires stim- ulation of SA (but not RA) afferents, and removal of descending inhibition reveals that their thresholds are relatively low (Weng and Schouenborg, 1998). In view of their peripheral physiology and central anatomy, HTMRs are not only in a position to play a prominent role in the afferent limb of these reflexes during normal postnatal development but also represent intuitive candidates from a functional perspective. The postnatal decrease in hypersensitivity may reflect predominantly functional maturation of descending inhibitory inputs (Fitzgerald and Koltzenburg, 1986), although possible alterations in the peripheral sensitivity and/or synaptic efficacy of one or more subsets of myelinated HTMRs may also play a role.

\section{References}

Baccaglini PI (1978) Action potentials of embryonic dorsal root ganglion neurones in Xenopus tadpoles. J Physiol (Lond) 283:585-604.

Burgess PR, Perl ER (1967) Myelinated afferent fibres responding specifically to noxious stimulation of the skin. J Physiol (Lond) 90:541-562.

Coggeshall RE, Jennings EA, Fitzgerald M (1996) Evidence that large myelinated primary afferent fibers make synaptic contacts in lamina II of neonatal rats. Dev Brain Res 92:81-90.

Djouhri L, Lawson SN (2001) Differences in the size of the somatic action potential overshoot between nociceptive and non-nociceptive dorsal root ganglion neurones in the guinea-pig. Neuroscience 108:479-491.

Djouhri L, Bleazard K, Lawson SN (1998) Association of somatic action potential shape with sensory receptive properties in guinea-pig dorsal root ganglion neurones. J Physiol (Lond) 513:857-872.

Fitzgerald M (1987) Cutaneous primary afferent properties in the hindlimb of the neonatal rat. J Physiol (Lond) 383:79-92.

Fitzgerald M, Fulton BP (1992) The physiological properties of developing sensory neurons. In: Sensory neurons: diversity, development and plasticity (Scott SA, ed), pp 287-306. New York: Oxford.

Fitzgerald M, Jennings E (1999) The postnatal development of spinal sensory processing. Proc Natl Acad Sci USA 96:7719-7722.

Fitzgerald M, Koltzenburg M (1986) The functional development of descending inhibitory pathways in the dorsolateral funiculus of the newborn rat spinal cord. Brain Res 389:261-270.

Fitzgerald M, Butcher T, Shortland P (1994) Developmental changes in the laminar termination of A fiber cutaneous sensory afferents in the rat spinal cord dorsal horn. J Comp Neurol 348:225-233.

Fulton BP (1987) Postnatal changes in conduction velocity and soma action potential parameters of rat dorsal root ganglion neurones. Neurosci Lett 73:125-130.

Fyffe REW (1992) Laminar organization of primary afferent terminations in the mammalian spinal cord. In: Sensory neurons: diversity, development and plasticity (Scott SA, ed), pp 131-139. New York: Oxford.

Hayashi H (1985) Morphology of terminations of small and large myelinated trigeminal primary afferent fibers in the cat. J Comp Neurol 240:71-89.

Hoheisel U, Lehmann-Willenbrock E, Mense S (1989) Termination patterns of identified group II and III afferent fibres from deep tissues in the spinal cord of the cat. Neuroscience 28:495-507.

Holmberg H, Schouenborg J (1996) Postnatal development of the nociceptive withdrawal reflexes in the rat: a behavioural and electromyographic study. J Physiol (Lond) 493:239-252.

Koerber HR, Mendell LM (1992) Functional heterogeneity of dorsal root ganglion cells. In: Sensory neurons: diversity, development and plasticity (Scott SA, ed), pp 77-96. New York: Oxford.

Koerber HR, Druzinsky RE, Mendell LM (1988) Properties of somata of spinal dorsal root ganglion cells differ according to peripheral receptor innervated. J Neurophysiol 60:1584-1596.

Koltzenburg M, Stucky CL, Lewin GR (1997) Receptive properties of mouse sensory neurons innervating hairy skin. J Neurophysiol 78:1841-1850.

Light AR, Perl ER (1979) Spinal terminations of functionally identified primary afferent neurons with slowly conducting myelinated fibers. J Comp Neurol 186:133-150.

Mirnics K, Koerber HR (1997) Properties of individual embryonic primary afferents and their spinal projections in the rat. J Neurophysiol 78:1590-1600.

Nakatsuka T, Ataka T, Kumamoto E, Tamaki T, Yoshimura M (2000) Alteration in synaptic inputs through C-afferent fibers to substantia gelati- 
nosa neurons of the rat spinal dorsal horn during postnatal development. Neuroscience 99:549-556.

Ogata N, Tatebayashi H (1992) Ontogenic development of the TTXsensitive and TTX-insensitive $\mathrm{Na}^{+}$channels in neurons of the cat dorsal root ganglia. Brain Res Dev Brain Res 65:93-100.

Park JS, Nakatsuka T, Nagata K, Higashi H, Yoshimura M (1999) Reorganization of the primary afferent termination in the rat spinal dorsal horn during postnatal development. Brain Res Dev Brain Res 113:29-36.

Perl ER (1968) Myelinated afferent fibres innervating the primate skin and their response to noxious stimuli. J Physiol (Lond) 197:593-615.

Rethelyi M, Light AR, Perl ER (1982) Synaptic complexes formed by functionally defined primary afferent units with fine myelinated fibers. J Comp Neurol 207:381-393.

Rifkin JT, Todd VJ, Anderson LW, Lefcort F (2000) Dynamic expression of neurotrophin receptors during sensory neuron genesis and differentiation. Dev Biol 227:465-480.

Ritter AM, Mendell LM (1992) Somal membrane properties of physiologically identified sensory neurons in the rat: effects of nerve growth factor. J Neurophysiol 68:2033-2041.

Ritter AM, Woodbury CJ, Albers KM, Davis BM, Koerber HR (2000) Maturation of cutaneous sensory neurons from normal and NGFoverexpressing mice. J Neurophysiol 83:1722-1732.

Rose RD, Koerber HR, Sedivec MJ, Mendell LM (1986) Somal action potential duration differs in identified primary afferents. Neurosci Lett 63:259-264.
Scheibel ME, Scheibel AB (1968) Terminal axon patterns in cat spinal cord. II. The dorsal horn. Brain Res 9:32-58.

Scott SA (1992) Sensory neurons: diversity, development and plasticity. New York: Oxford.

Tong YG, Wang HF, Ju G, Grant G, Hökfelt T, Zhang Z (1999) Increased uptake and transport of cholera toxin B-subunit in dorsal root ganglion neurons after peripheral axotomy: possible implications for sensory sprouting. J Comp Neurol 404:143-158.

Traub RJ, Mendell LM (1988) The spinal projection of individual identified A-delta- and C-fibers. J Neurophysiol 59:41-55.

Weng HR, Schouenborg J (1998) On the cutaneous receptors contributing to withdrawal reflex pathways in the decerebrate spinal rat. Exp Brain Res 118:71-77.

Woodbury CJ, Ritter AM, Koerber HR (2000) On the problem of lamination in the superficial dorsal horn of mammals: a re-appraisal of the substantia gelatinosa in postnatal life. J Comp Neurol 417:88-102.

Woodbury CJ, Ritter AM, Koerber HR (2001) Central anatomy of individual rapidly adapting low-threshold mechanoreceptors innervating the "hairy" skin of newborn mice: early maturation of hair follicle afferents. J Comp Neurol 436:304-323.

Woolf CJ, Shortland P, Coggeshall RE (1992) Peripheral nerve injury triggers central sprouting of myelinated afferents. Nature 355:75-78.

Ziskind-Conhaim L (1988) Physiological and morphological changes in developing peripheral nerves of rat embryos. Brain Res 470:15-28. 